\title{
AMPLIFIED SPONTANEOUS EMISSION IN RHODAMINE DYES: GENERATION OF PICOSECOND LIGHT PULSES AND DETERMINATION OF EXCITED STATE ABSORPTION AND RELAXATION
}

\author{
W. FALKENSTEIN, A. PENZKOFER * and W. KAISER \\ Physik Department der Technischen Universität München, \\ München, Federal Republic Germany
}

Received 12 July 1978

\begin{abstract}
The following quantitative investigations with picosecond pump pulses were made as a function of intensity: (1) fluorescence in the forward direction; (2) duration of the fluorescence; (3) and (4) spectral peak and width of the emission; (5) energy transmission of the pump pulse. Large energy amplification (factor 300) and drastic reduction of the duration of the fluorescence (factor 150) were observed. Comparison with model calculations allows the determination of various molecular absorption and relaxation parameters.
\end{abstract}

During the past decade, amplified spontaneous emission of fluorescent molecules has been studied under a variety of experimental conditions [1]. While stimulated emission is the prerequisite for any laser, the same physical process might be quite disturbing when spectroscopic parameters (e.g. the spontaneous emission lifetime) are measured at high excitation intensities. Qualitative investigations of amplified spontaneous emission of organic dyes have been reported by a number of authors using picosecond light pulses as pump source [2-6].

In this letter we present quantitative measurements of five physical phenomena studied as a function of input intensity with molecular concentration as the main parameter. We investigated; (1) the fluorescence emission in the forward direction; (2) the reduced duration of the fluorescence; (3) the wavelength shift of the peak of the emission, (4) the spectral width of the fluorescence and (5) the energy transmission of the picosecond pump pulse. The purpose of these investigations is two-fold: First, we wanted to see to which extent the fluorescence emission changes for high input intensities; in particular we were interested in the shortest possible pulse duration of the fluorescence

\footnotetext{
* Present address: Universität Regensburg, Germany
}

emission. Second, we determined important molecular absorption and relaxation parameters by fitting model calculations to our various experimental results.

Experimentally we worked with a mode-locked Ndglass laser [7]. A single pulse is selected from the beginning of the pulse train with an electro-optical shutter. The second harmonic $\left(\widetilde{\nu}_{\mathrm{L}}=18910 \mathrm{~cm}^{-1}\right)$ of the laser pulse is generated in a KDP crystal. The duration and width of the green pulse is $\Delta t_{\mathrm{L}} \approx 4 \mathrm{ps}$ and $\Delta \widetilde{\nu}$ $\approx 5 \mathrm{~cm}^{-1}$, respectively. The peak intensity is determined by measuring the energy transmission through a two-photon absorbing rutile crystal [8]. Rhodamine $6 \mathrm{G}$ and rhodamine $\mathrm{B}$ dissolved in ethanol are investigated. The dye cells are tilted to avoid amplification of reflected fluorescence light. Different detection systems were applied after the sample depending upon the specific investigation. The divergence of the amplified spontaneous emission was analysed with apertures of different size. The fluorescence energy within a solid angle $\Delta \Omega$ was measured with a photomultiplier. The fluorescence duration was recorded with a streak camera (3ps resolution). The spectral distribution of the fluorescence light, the spectral narrowing, and the spectral shift were studied with a spectrometer in conjunction with an optical multi-channel analyser.

(1) The normalized fluorescence energy $\bar{E}_{\mathrm{F}}=E_{\mathrm{F}} / E_{\mathrm{Abs}}$ 
versus the peak intensity $I_{0 \mathrm{~L}}$ of the pump pulse is shown in fig. 1. $E_{\mathrm{F}}$ represents the fluorescence energy emitted in forward direction within a solid angle $\Delta \Omega_{\text {exp }}=1.40 \times 10^{-3}$ sr. $E_{\text {Abs }}$ is the laser energy absorbed in the dye sample. The points represent experimental data and the curves are calculated (see below). The open circles (curves 1) and the triangles (curves 2) correspond to dye concentrations of $10^{-4} \mathrm{M}$ and 2 $\times 10^{-4} \mathrm{M}$, respectively. The effective length of the tilted samples is $1.13 \mathrm{~cm}$. The fluorescence at low intensities $\left(I_{0 \mathrm{~L}} \leqslant 5 \times 10^{8} \mathrm{~W} / \mathrm{cm}^{2}\right)$ is determined by spontaneous emission. Fig. 1 shows clearly the rapid rise of the fluorescence emission resulting from stimulated emission $\left(I_{0 \mathrm{~L}}>10^{9} \mathrm{~W} / \mathrm{cm}^{2}\right)$. At very high pump intensities the normalized emission levels or decreases as a result of excited state absorption. At lower dye concentrations of $4 \times 10^{-5} \mathrm{M}$ in cells of $0.56 \mathrm{~cm}$ length an increase of $\bar{E}_{\mathrm{F}}$ could not be observed. The number of inverted molecules is too small for effective amplification. At very high concentrations $(\gtrsim 4$ $\left.\times 10^{-4} \mathrm{M}\right)$, on the other hand, the input pulse is ab-

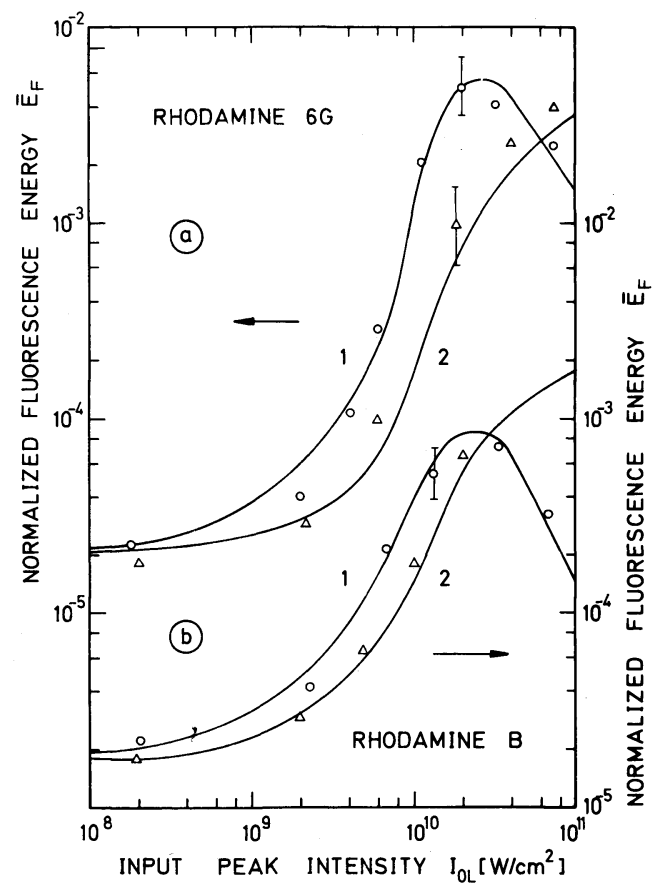

Fig. 1. Normalized fluorescence energy $\bar{E}_{\mathrm{F}}$ versus input peak intensity for (a) rhodamine $6 \mathrm{G}$ and (b) rhodamine B. Experimental points: circles, $C=10^{-4} \mathrm{M}$; triangles, $C=2 \times 10^{-4} \mathrm{M}$. The curves are calculated using the data of table 1 . sorbed at the very beginning of the sample and the forward direction is not preferred in the amplification process.

(2) The duration of the fluorescence pulses was determined from streak camera traces of the forward emitted fluorescence light. The rise time of the fluorescence emission was found to be approximately $6 \mathrm{ps}$ in agreement with the length of the pump pulse. Following the excitation, the fluorescence signal decays nonexponentially due to the fast depopulation of the $S_{1}$ level by amplified spontaneous emission. At later times, when the $S_{1}$ population is small, the normal spontaneous emission rate is observed.

In fig. 2 the duration $\Delta t_{\mathrm{F}}$ (fwhm) of the fluorescence is presented. The dye concentrations are $10^{-4} \mathrm{M}$ (circles, curves 1 ) and $2 \times 10^{-4} \mathrm{M}$ (triangles, curves 2 ). Fluorescence emission as short as 20 ps was experimentally observed. Comparable fluorescence shortening was observed with $4 \times 10^{-4}$ molar solutions. The curves are calculated with the same parameters as used in fig. 1.

(3) The absorption and emission bands overlap and the short wavelength fluorescence is reabsorbed. The observed fluorescence peak is shifted to longer wavelengths for higher dye concentrations. With increased pump intensity, however, the ground state absorption is reduced and the fluorescence emission shifts to

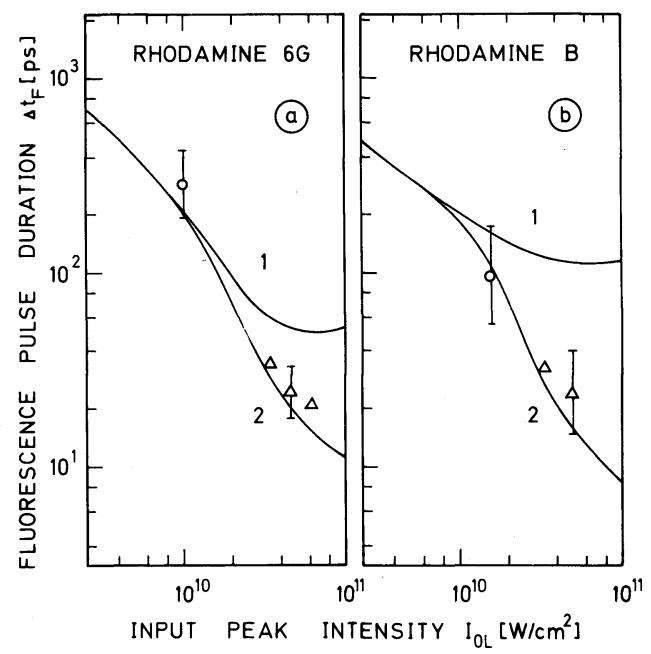

Fig. 2. Duration of amplified spontaneous emission versus input peak intensity for (a) rhodamine $6 \mathrm{G}$ and (b) rhodamine B. Dye concentrations $10^{-4} \mathrm{M}$ (circles, curves 1 ), and 2 $\times 10^{-4} \mathrm{M}$ (triangles, curves 2 ). The curves are calculated with the same parameters as in fig. 2 . 
shorter wavelengths, i.e. towards the frequency of the peak stimulated emission cross section $\sigma_{\mathrm{em}}$ (see figs. 3a, $3 b$ and 6$)$.

(4) The spectral width of the fluorescence decreases at high pump intensities. The amplification of spontaneous emission depends on the spectral distribution of the emission cross section $\sigma_{\mathrm{em}}(\nu)$ leading to a spectral narrowing of the fluorescence light. For $10^{-4} \mathrm{M}$ rhodamine $6 \mathrm{G}$ the experimentally observed spectral width is depicted in fig. 3c. At low intensities a half width of $\sim 1050 \mathrm{~cm}^{-1}$ is observed. For high pump intensities the spectral width reduces to $\sim 320 \mathrm{~cm}^{-1}$. Theoretical studies [9] predict for inhomogeneously broadened lines at high gain:

$$
\begin{aligned}
& \Delta \nu_{\mathrm{F}}\left(I_{\mathrm{L}}\right) / \Delta \nu_{\mathrm{F}}(0) \\
& \quad=\left(\ln \left\{\bar{E}_{\mathrm{F}}\left(I_{\mathrm{L}}\right) \Delta t_{\mathrm{F}}(0) /\left[\bar{E}_{\mathrm{F}}(0) \Delta t_{\mathrm{F}}\left(I_{\mathrm{L}}\right)\right]\right\}\right)^{-1 / 2}
\end{aligned}
$$

In fig. $3 \mathrm{c}$ the amplification has a value of $\bar{E}_{\mathrm{F}}\left(I_{\mathrm{L}}\right) / \bar{E}_{\mathrm{F}}(0)$

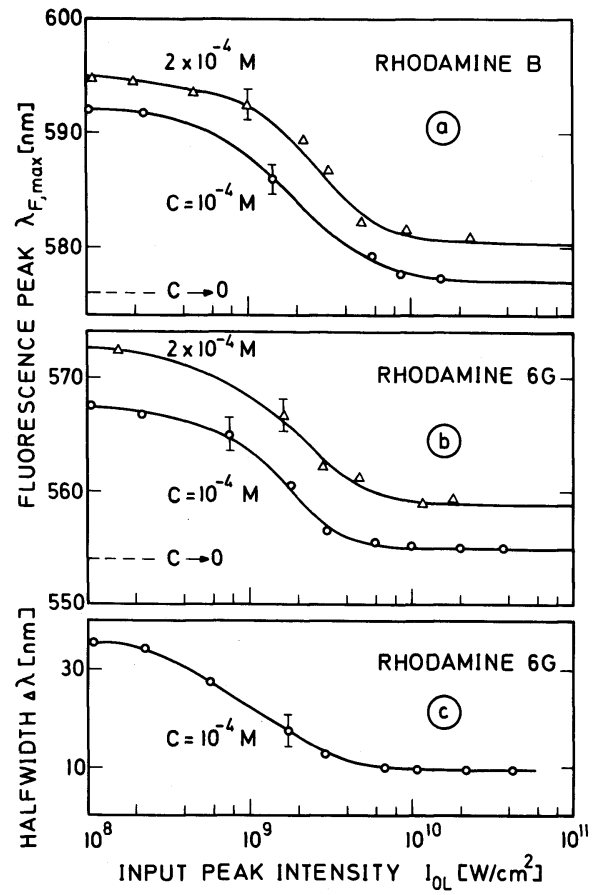

Fig. 3. Shift of spectral peak of fluorescence emission of rhodamine $\mathrm{B}$ (a) and rhodamine $6 \mathrm{G}$ (b) and spectral narrowing of rhodamine $6 \mathrm{G}$ (c) versus input peak intensity. The wavelength of peak fluorescence emission at low dye concentrations is indicated by the dashed lines. $\approx 300$ and the pulse shortening was measured to be $\Delta t_{\mathrm{F}}(0) / \Delta t_{\mathrm{F}}\left(I_{\mathrm{L}}\right) \approx 30$ at high pump intensities. With these numbers we calculate a frequency narrowing of $\Delta \nu_{\mathrm{F}}\left(I_{\mathrm{L}}\right) / \Delta \nu_{\mathrm{F}}(0)=0.33$ which is in good agreement with the experimental value of 0.30 .

(5) The energy transmissions through rhodamine $6 \mathrm{G}$ and rhodamine $\mathrm{B}$ are depicted in figs. $4 \mathrm{a}$ and $\mathrm{b}(l$ $\left.=13 \mathrm{~cm}, C=10^{-4} \mathrm{M}\right)$. The circles and triangles represent experimental data for $\Delta t_{\mathrm{L}}=4 \mathrm{ps}$ and $20 \mathrm{ps}$, respectively. The data points indicate a rapid bleaching of the solution. The transmission depends on the excited state absorption and on the relaxation from higher excited states as analysed below.

(6) Using mixtures of rhodamine $6 \mathrm{G}$ and rhodamine $B$ the frequency of the fluorescence emission peak could be shifted continuously between the emission frequencies of the pure dye solutions, i.e. between 555 and $580 \mathrm{~nm}$. This fact allows the generation of picosecond light pulses with tunable frequencies.

In a theoretical model we described the dissolved rhodamine molecules by a multi-level system contain-

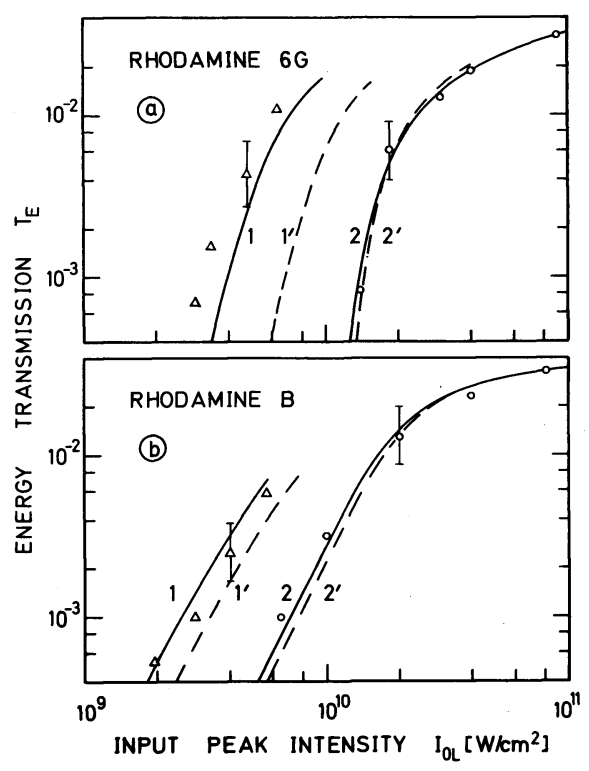

Fig. 4. Energy transmission through rhodamine samples. $l$ $=1.13 \mathrm{~cm}, C=10^{-4} \mathrm{M}$; curves 1 and $1^{\prime}, \Delta t_{\mathrm{L}}=20 \mathrm{ps}$ (triangles); curves 2 and $2^{\prime}, \Delta t_{\mathrm{L}}=4 \mathrm{ps}$ (circles). (a) Rhodamine $6 \mathrm{G}$. Solid curves $(1$ and 2$), \sigma_{\text {exL }}=5 \times 10^{-17} \mathrm{~cm}^{2}$ and $k_{6}=10^{13} \mathrm{~s}^{-1}$; dashed curves $\left(1^{\prime}\right.$ and $\left.2^{\prime}\right), \sigma_{\mathrm{exL}}=7.5 \times 10^{-12} \mathrm{~cm}^{2}$ and $k_{6}=10^{17} \mathrm{~s}^{-1}$.(b) Rhodamine B. Solid curves, $\sigma_{\text {exL }}=5$ $\times 10^{-17} \mathrm{~cm}^{2}$ and $k_{6}=10^{13} \mathrm{~s}^{-1}$; dashed curves, $\sigma_{\text {exL }}$ $=6 \times 10^{-17} \mathrm{~cm}^{2}$ and $k_{6}=10^{12} \mathrm{~s}^{-1}$. 


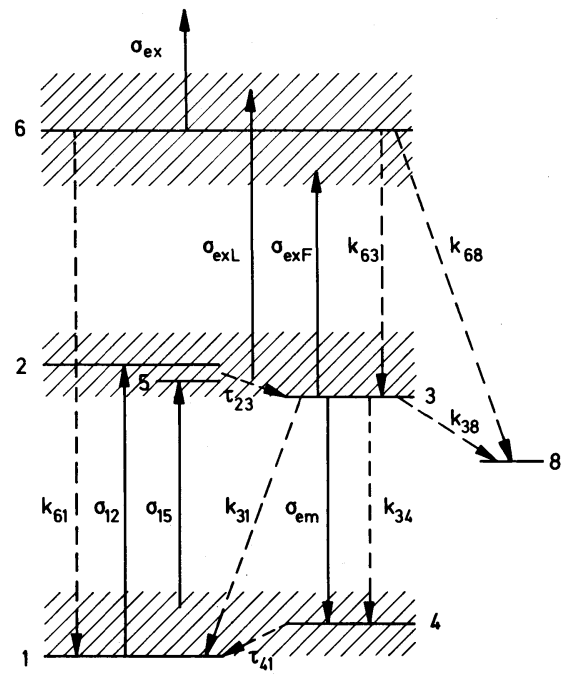

Fig. 5. Multi-level model. 1, ground state; 2, Franck-Condon state in $\mathrm{S}_{1}$-band; 3 , temporal equilibrium position in $\mathrm{S}_{1}$-band; 4, Franck-Condon state in $\mathrm{S}_{0}$-band; 5 , state in $\mathrm{S}_{1}$-band reached by reabsorption; $6, S_{n}$-state populated by excited state absorption; 8 , triplet state or state of photodecomposed molecules. The solid arrows indicate absorption and stimulated emission processes, the dashed arrows illustrate the various relaxation channels.

ing different absorption, emission and relaxation processes [10] (see fig. 5). Rate equations for the energy states and for the photon fluxes of the pump and fluorescence radiation were solved on a computer. The considered energy levels are: 1 , ground state; 2 , pumped Franck-Condon state in the $S_{1}$ band; 3 , temporal equilibrium level in the $S_{1}$ state; 4 , Franck-Condon level in the ground state $S_{0} ; 5$, level in the $S_{1}$ band populated by reabsorption of fluorescence light; $6, S_{n}$-level populated by excited state absorption of laser $\left(\sigma_{\text {exL }}\right)$ and fluorescence light $\left(\sigma_{\mathrm{exF}}\right) ; 8$, level comprising triplet and decomposition states.

Model calculations allow us to estimate the excited state absorption cross-sections and relaxation rates. These data are listed together with known parameters in table 1:

(i) $\sigma_{12}$ is the average absorption cross-section of isotropically distributed dye molecules in the ground state. The molecular dichroism $D_{\mathrm{m}}=\left(\sigma_{\|}-\sigma_{\perp}\right) /\left(\sigma_{\|}+\sigma_{\perp}\right)$ represents the dependence of absorption on the orientation of molecules. $\left(\sigma_{12}=\left(\sigma_{\|}+2 \sigma_{\perp}\right) / 3 ; \sigma_{\|}=3 \sigma_{12}(1\right.$ $\left.\left.+D_{\mathrm{m}}\right) /\left(3-D_{\mathrm{m}}\right) ; \sigma_{\perp}=3 \sigma_{12}\left(1-D_{\mathrm{m}}\right) /\left(3-D_{\mathrm{m}}\right)\right)[11$, $12] . D_{\mathrm{m}}$ is assumed to be equal for the ground state and the excited state absorption [12].

(ii) The cross-section of stimulated emission $\sigma_{\mathrm{em}}$ is strongly wavelength dependent. The $\sigma_{\mathrm{em}}(\lambda)$-curves are obtained from the spectral distribution $E(\lambda)$ of the fluorescence light (quantum distribution; $\int E(\lambda) \mathrm{d} \lambda$ $=1)$. The relation between $\sigma_{\mathrm{em}}(\lambda)$ and $E(\lambda)$ is [13]

$$
\sigma_{\mathrm{em}}(\lambda)=\lambda^{4} k_{34} E(\lambda) / 8 \pi \eta^{2} c
$$

\begin{tabular}{|c|c|c|c|c|}
\hline Parameter & Rhodamine 6G & & Rhodamine B & \\
\hline$\sigma_{12}\left(\widetilde{v}_{\mathrm{I}}=18910 \mathrm{~cm}^{-1}\right)$ & $4.17 \times 10^{-16} \mathrm{~cm}^{2}$ & [11] & $1.87 \times 10^{-16} \mathrm{~cm}^{2}$ & [11] \\
\hline$D_{\mathrm{m}}$ & $0.95 \pm 0.03$ & {$[12]$} & $0.93 \pm 0.03$ & {$[12]$} \\
\hline$\tau_{23}$ & $(0.9 \pm 0.3) \mathrm{ps}$ & [11] & $(1.1 \pm 0.3) \mathrm{ps}$ & [11] \\
\hline$\tau_{41}$ & $\approx 1 \mathrm{ps}$ & & $\approx 1 \mathrm{ps}$ & \\
\hline$k_{31}$ & $(2.8 \pm 0.3) \times 10^{7} \mathrm{~s}^{-1}$ & {$[17,18]$} & $(1.85 \pm 0.2) \times 10^{8} \mathrm{~s}^{-1}$ & [19] \\
\hline$k_{34}$ & $(2.1 \pm 0.2) \times 10^{8} \mathrm{~s}^{-1}$ & {$[17,18, \mathrm{a}]$} & $(1.85 \pm 0.2) \times 10^{8} \mathrm{~s}^{-1}$ & {$[19, \mathrm{a}]$} \\
\hline$k_{38}$ & $4.2 \times 10^{5} \mathrm{~s}^{-1}$ & {$[20]$} & $1.7 \times 10^{6} \mathrm{~s}^{-1}$ & {$[18]$} \\
\hline \multicolumn{5}{|l|}{ results obtained here } \\
\hline$\sigma_{\mathrm{exL}}$ & \multirow{2}{*}{\multicolumn{2}{|c|}{$\begin{array}{l}(5 \pm 1) \times 10^{-17} \mathrm{~cm}^{2} \\
(7 \pm 2) \times 10^{-17} \mathrm{~cm}^{2}\end{array}$}} & \multicolumn{2}{|l|}{$(5 \pm 1) \times 10^{-17} \mathrm{~cm}^{2}$} \\
\hline$\sigma_{\mathrm{exF}}$ & \multirow{2}{*}{\multicolumn{2}{|c|}{$\begin{array}{l}(7 \pm 2) \times 10^{-17} \mathrm{~cm}^{2} \\
\geqslant 2 \times 10^{12} \mathrm{~s}^{-1}\end{array}$}} & \multirow{2}{*}{\multicolumn{2}{|c|}{$\begin{array}{l}(1.0 \pm 0.3) \times 10^{-16} \mathrm{~cm}^{2} \\
\geqslant 10^{13} \mathrm{~s}^{-1}\end{array}$}} \\
\hline$k_{6}$ & & & & \\
\hline$k_{61} / k_{6}$ & \multicolumn{2}{|l|}{$0.05 \pm 0.05$} & \multicolumn{2}{|l|}{$0.3 \pm 0.05$} \\
\hline$k_{63} / k_{6}$ & \multicolumn{2}{|l|}{$0.95 \pm 0.05$} & \multicolumn{2}{|l|}{$0.7 \pm 0.05$} \\
\hline$k_{68} / k_{6}$ & \multicolumn{2}{|l|}{$0.01 \pm 0.01$} & \multicolumn{2}{|l|}{$0.02 \pm 0.01$} \\
\hline
\end{tabular}

\footnotetext{
a Values redetermined in this paper.
} 
where $\eta$ is the refractive index at wavelength $\lambda$ and $c$ the light velocity. In our measurements we determined $E(\lambda)$ with a spectrograph and an optical multichannel analyser at a dye concentration of $2 \times 10^{-6} \mathrm{M}$. The $\sigma_{\mathrm{em}}(\lambda)$-curves are depicted in fig. 6. (Earlier reports on $\sigma_{\text {em }}(\lambda)$ used unaccurate $k_{34}$-values leading to somewhat different results).

(iii) The relaxation $\tau_{23}$ within the $S_{1}$-state was measured to approximately $1 \mathrm{ps}$ [11]. The corresponding relaxation time $\tau_{41}$ in the $S_{0}$-state is assumed to be similar [14].

(iv) The transition rates $k_{31}, k_{34}$ and $k_{38}$ are determined by measuring the low-intensity fluorescence decay time $\bar{\tau}_{\mathrm{F}}=1 /\left(k_{31}+k_{34}+k_{38}\right)=1 / k_{3}$, the fluorescence quantum yield $q_{\mathrm{F}}=k_{34} / k_{3}$ and the intersystem crossing efficiency $q_{\mathrm{ISC}}=k_{38} / k_{3}$. We remeasured the fluorescence decay times at $C=2 \times 10^{-6} \mathrm{M}$ with fast photomultipliers and found $\tau_{\mathrm{F}}=(4.2 \pm 0.3) \mathrm{ns}$ and $(2.7 \pm 0.3)$ ns for rhodamine $6 \mathrm{G}$ and rhodamine $\mathrm{B}$, respectively.

We recall that the radiation energy $E_{\mathrm{F}}$ was measured through a constant aperture. The amplified fluorescence emission however occurs within a larger solid angle $\Delta \Omega_{1 / 2}$ given by [10]

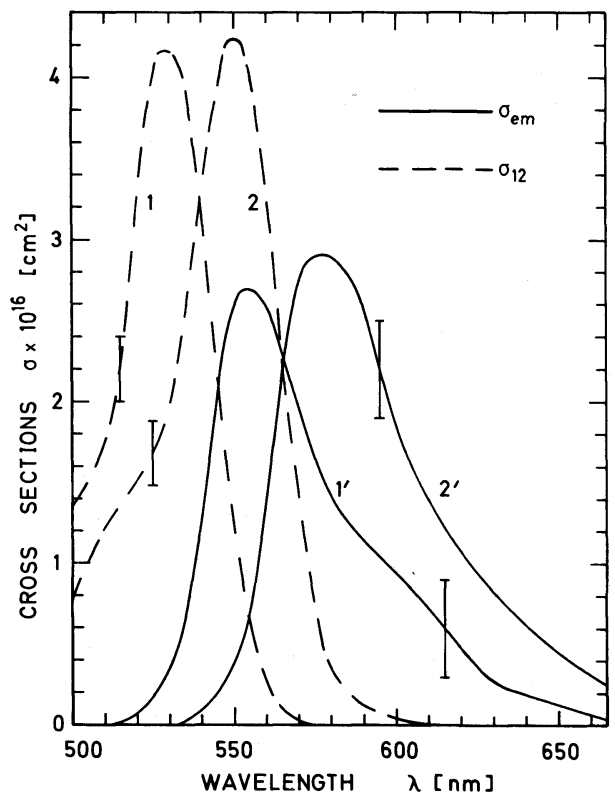

Fig. 6. Absorption and emission spectra for rhodamine $6 \mathrm{G}$ (curves 1 and $1^{\prime}$ ) and rhodamine B (curves 2 and $2^{\prime}$ ).
$\Delta \Omega_{1 / 2}=\frac{\pi d_{\mathrm{L}}^{2}}{4 l_{\text {eff }}^{2}} \frac{\ln \kappa-\ln \ln \{[\exp (\kappa)+1] / 2\}}{\ln 2}$,

where $d_{\mathrm{L}} \approx 0.5 \mathrm{~mm}$ is the diameter of the pump beam, $l_{\text {eff }}$ is the penetration depth of the laser light and $\kappa$ $=\ln \left\{\left[\bar{E}_{\mathrm{F}}\left(I_{\mathrm{L}}\right) / \Delta t_{\mathrm{F}}\left(I_{\mathrm{L}}\right)\right] /\left[\bar{E}_{\mathrm{F}}(0) / \Delta t_{\mathrm{F}}(0)\right]\right\}$ represents the narrowing at high intensities. $\Delta \Omega_{1 / 2}$ depends strongly on the dye concentration and the pump intensity. The calculated solid angles agree with experimental observations.

The average reabsorption cross section $\sigma_{15}$ is approximately equal to the ground state absorption crosssection at the emitting wavelength $\sigma_{12}\left(\lambda_{\mathrm{F} \text {, max }}\right)$. The $\sigma_{12}(\lambda)$ curves are included in fig. 6 and $\lambda_{F, \max }$ is depicted in fig. 3a and b. At low pump intensities the fluorescence emission is quite broad and the absorption cross section varies substantially over the fluorescence band width. By comparing the experimental fluorescence signals (at $I_{0 \mathrm{~L}} \approx 10^{8} \mathrm{~W} / \mathrm{cm}^{2}$ ) with calculation we find effective $\sigma_{15}$-values of $(2 \pm 0.2)$ $\times 10^{-17} \mathrm{~cm}^{2}$ and $(1 \pm 0.2) \times 10^{-17} \mathrm{~cm}^{2}$, respectively, for $10^{-4} \mathrm{M}$ and $2 \times 10^{-4} \mathrm{M}$ solutions of rhodamine $6 \mathrm{G}$ and rhodamine $\mathrm{B}$.

We now wish to compare our experimental data with calculated results and determine various molecular parameters. In particular we are interested in the excited state absorption cross-sections $\sigma_{\mathrm{exL}}$ and $\sigma_{\mathrm{exF}}$ and the relaxation rates $k_{61}, k_{63}$ and $k_{68}$.

(1) The energy transmission $T_{\mathrm{E}}$ at high pump intensities is used to determine $\sigma_{\text {exL }}$ and $k_{6} . T_{\mathrm{E}}$ depends on the known parameters $\sigma_{12}$ and $\tau_{23}[11,12]$ and on $\sigma_{\text {exL }}$ and $k_{6}$. The effect of $k_{6}$ on $T_{\mathrm{E}}$ results from the fact that for $k_{6} \lesssim \Delta t_{\mathrm{L}}^{-1}$ the molecules accumulate in level 6 and reduce the excited state absorption, while for $k_{6}>\Delta t_{\mathrm{L}}^{-1}$ the molecules return to the initial states and increase the absorption. A measurement of $T_{\mathrm{E}}$ for two different pulse durations (different ratios of $\left.k_{6} / \Delta t_{\mathrm{L}}^{-1}\right)$ allows the separate determination of $\sigma_{\text {exL }}$ and $k_{6}$.

In fig. $4 \mathrm{a}$ and $\mathrm{b}$ the experimental results are presented together with calculated curves. Curves 1 and $1^{\prime}$ belong to $\Delta t_{\mathrm{L}}=20 \mathrm{ps}$ (triangles) while curves 2 and $2^{\prime}$ respond to $\Delta t_{\mathrm{L}}=4 \mathrm{ps}$ (circles). The solid curves 1 and 2 , calculated with the same $\sigma_{\text {exL }}$ and $k_{6}$ values, agree with the experimental points at both durations. For other sets of $\sigma_{\text {exL }}$ and $k_{6}$ a simultaneous fit at $\Delta t_{\mathrm{L}}=4 \mathrm{ps}$ and $20 \mathrm{ps}$ is not possible as the dashed curves indicate. 
The $\sigma_{\text {exL }}$ and $k_{6}$ values estimated from fig. 4 are listed in table 1 .

(2) The branching ratio $k_{63} / k_{6}$ was determined by comparing the quantum yields of the $S_{1}-S_{0}$ fluorescence after excitation with light at $\lambda=265 \mathrm{~nm}$ and at $\lambda=530 \mathrm{~nm}$. Equal quantum yields would indicate that all highly excited molecules in level 6 return to level 3 , i.e. $k_{63}=k_{6}$. In the measurements we compare two fluorescence signals taking into account the absorbed photon energy. The fluorescence life time $\tau_{\mathrm{F}}$ was found to be the same for both excitation wavelengths. The observed $\mathrm{S}_{1}-\mathrm{S}_{0}$ quantum yields give $k_{63} / k_{6}$ $=0.95 \pm 0.05$ and $0.7 \pm 0.05$ for rhodamine $6 \mathrm{G}$ and rhodamine $\mathrm{B}$, respectively $\left(C=2 \times 10^{-6} \mathrm{M}\right)$. The relaxation rate of rhodamine molecules from $\lambda_{\text {exc }}$ $\approx 307.5 \mathrm{~nm}$ to $\mathrm{S}_{1}$ was studied in [16] and found to be $\geqslant 5 \times 10^{12} \mathrm{~s}^{-1}$.

(3) The transition rate $k_{68}$ leads to a loss of fluorescing molecules; it reduces the amplification and the pulse shortening of fluorescence light at high pump intensities. In our experiments, the $k_{68} / k_{6}$ ratio was determined by adjusting the calculated curves 1 of fig. $1 \mathrm{a}$ and b at $I_{0 \mathrm{~L}}>3 \times 10^{10} \mathrm{~W} / \mathrm{cm}^{2}$ to the decreasing experimental $\bar{E}_{\mathrm{F}}$-values. The obtained results of $k_{68} / k_{6}=0.01 \pm 0.01$ for rhodamine $6 \mathrm{G}$ and 0.02 \pm 0.01 for rhodamine $B$ indicate that the loss of molecules from the singlet system is small.

(4) The ratio $k_{61} / k_{6}$ of molecules returning directly to the ground state is found by the relation $k_{61} / k_{6}$ $=1-k_{63} / k_{6}-k_{68} / k_{6}$. The obtained values for rhodamine $6 \mathrm{G}$ and rhodamine $\mathrm{B}$ are $0.05 \pm 0.05$ and 0.3 \pm 0.05 , respectively.

(5) The excited state absorption of the pump laser $\sigma_{\text {exL }}$ and the fluorescence light $\sigma_{\text {exF }}$ reduces the amplification of spontaneous emission. Having determined $\sigma_{\text {exL }}$ (see above) we estimated $\sigma_{\text {exF }}$ by fitting curves 1 of fig. $1 \mathrm{a}$ and $\mathrm{b}$ to the experimental points in the range between $5 \times 10^{9}$ and $2 \times 10^{10} \mathrm{~W} / \mathrm{cm}^{2}$. The obtained $\sigma_{\mathrm{exF}}$-values are somewhat larger than $\sigma_{\text {exL }}$ (see table 1). In our calculations absorption from level 6 to high states is included by assuming the same absorption coefficients as for the $\mathrm{S}_{1}$-level.
In this paper we demonstrated the generation of picosecond light pulses at new frequencies by amplified spontaneous emission. With the help of model calculations we determined several excited state parameters of the rhodamines.

The authors thank Professors A. Laubereau and M. Maier for helpful discussions.

\section{References}

[1] U. Ganiel, A. Hardy, G. Neumann and D. Treves, IEEE J. Quant. Electron. QE-11 (1975) 881.

[2] M.E. Mack, Appl. Phys. Letters 15 (1969) 166.

[3] M.M. Malley and P.M. Rentzepis, Chem. Phys. Letters 7 (1970) 57.

[4] C. Lin, T.K. Gustafson and A. Dienes, Opt. Comm. 8 (1973) 210.

[5] A.N. Rubinov, M.C. Richardson, K. Sala and A.J. Alcock, Appl. Phys. Letters 27 (1975) 358.

[6] G.R. Fleming, A.E.W. Knight, J.M. Morris, R.J. Robbins and G.W. Robinson, Chem. Phys. 23 (1977) 61.

[7] A. Laubereau and W. Kaiser, Opto-Electr. 6 (1974) 1.

[8] A. Penzkofer and W. Falkenstein, Opt. Comm. 17 (1976) 1.

[9] L.W. Casperson and A. Yariv, IEEE J. Quant. Electr. QE-8 (1972) 80.

[10] A. Penzkofer and W. Falkenstein, Opt. Quant. Electr. 10 (1978).

[11] A. Penzkofer, W. Falkenstein and W. Kaiser, Chem. Phys. Letters 44 (1976) 82.

[12] A. Penzkofer and W. Falkenstein, Chem. Phys. Letters 44 (1976) 547.

[13] O.G. Peterson, J.P. Webb, W.C. McColgin and J.H. Eberly, J. Appl. Phys. 42 (1971) 1917.

[14] D. Ricard and J. Ducuing, J. Chem. Phys. 62 (1975) 3616.

[15] H.B. Lin and M.R. Topp, Chem. Phys. Letters 47 (1977) 442.

[16] C.V. Shank, E.P. Ippen and O. Teschke, Chem. Phys. Letters 39 (1976) 320.

[17] D.W. Phillion, Appl. Phys. Letters 27 (1975) 85.

[18] B.G. Huth, G.I. Farmer and M.R. Kagen, J. Appl. Phys. 40 (1969) 5145.

[19] A.V. Aristov and Yu. S. Maslyukov, Opt. and Spectrosc. 41 (1976) 141.

[20] D.N. Dempster, J. Photochem. 2 (1973) 343. 